\title{
Lucas Sierra y los inicios de la Cirugía Biliar en Chile: a 130 años de la colecistectomía de Langenbuch*
}

\author{
Dr. RICARDO ESPINOZA G. ${ }^{1,2}$
}

Facultad de Medicina Universidad de los Andes.

2 Clínica Universidad de los Andes.

Santiago, Chile.

Abstract

\section{Lucas Sierra and early biliary surgery in Chile:130 year after Langenbuch cholecystectomy}

Through the communication of the first cholecystectomy performed in Chile by Dr. Lucas Sierra Mendoza in 1899, is reviewed the surgical setting of the time and highlights the features of this distinguished surgeon. Referring to Langenbuch initial experience, we illustrate the pathogenic conceptions of biliary gallstone disease and walk the path of cholecystectomy in the following century.

Key words: Cholecystectomy, pioneer, Lucas Sierra.

\section{Resumen}

A través de la comunicación de la primera colecistectomía efectuada en Chile por el doctor Lucas Sierra Mendoza en 1899, se revisa el contexto quirúrgico de la época y se destacan las características de este distinguido cirujano. Con referencia a la experiencia inicial de Langenbuch, se expone la concepción etiopatogénica de la enfermedad biliar litiásica y se recorre el camino de la colecistectomía en la centuria siguiente.

Palabras clave: Colecistectomía, pionero, Lucas Sierra.

Hace 130 años, exactamente en julio de 1882, en el Lazarus Krankenhaus de Berlín, su Director, el doctor Carl Johann August Langenbuch (1846-1901) realizó la primera colecistectomía ${ }^{1-3}$. Se había preparado por años, realizando disecciones en cadáveres $^{4}$. Su primer paciente fue un hombre de 43 años, portador de una colelitiasis sintomática, adicto a la morfina como consecuencia del uso repetido de esta droga ante las crisis de dolor, muy enflaquecido ${ }^{4,5}$. El paciente fue preparado por cinco días en base a enemas y aquel 15 de julio fue intervenido a través de una incisión subcostal extendida en T, a lo largo del borde lateral del músculo recto anterior derecho del abdomen. Expuesta la cavidad, Langenbuch extirpó una vesícula fibrosa, de paredes engrosadas, que contenía dos cálculos. Si bien su descripción quirúrgica no hacía alusión a la arteria cística, dejó claramente estipulado que el conducto cístico fue ligado con seda, y que para ese uso el catgut estaba absolutamente contraindicado ${ }^{3}$. La evolución postoperatoria de este primer paciente colecistectomizado cursó aparentemente sin complicaciones; al día

*Recibido el 23 de mayo de 2012 y aceptado para publicación el 27 de junio de 2012.

El autor no refiere conflictos de interés.

Correspondencia: Dr. Ricardo Espinoza G.

Av. San Carlos de Apoquindo 2200, Santiago, Chile.

respinoza@uandes.cl 
siguiente a la intervención no tenía dolor, se encontraba afebril y pudo fumar un cigarro. Se autorizó su levantada al día 12 y fue dado de alta al cumplir seis semanas en el hospital ${ }^{4}$. La primera colecistectomía de la historia fue publicada ese mismo año en el Berliner Klinische Wochenschrift ${ }^{5}$, cuyo detalle podemos apreciar en la Figura 1.

Al año siguiente, en el Congreso Alemán de Cirugía, Langenbuch dio cuenta de un total de tres pacientes exitosamente operados, formulando que esta cirugía estaba indicada porque la vesícula formaba los cálculos y los cirujanos no debían contentarse sólo con extraerlos ${ }^{6}$. De hecho, este nuevo procedimiento competía con la colecistostomía, que ya se aplicaba en forma regular desde hacía varios años, pero con una mayor mortalidad, que se situaba alrededor del 27\%. En cambio, la colecistectomía, sobre la evaluación de los primeros casos, exhibía una mortalidad de tan sólo un 12\%. Correspondió al mismo Langenbuch demostrar a la opinión mundial que la extirpación vesicular constituía una mejor alternativa terapéutica ${ }^{1,7,8}$; pocos años después, ya se habían realizado en el mundo cerca de 100 colecistectomías, con sobrevida en más del $80 \%$ de los pacientes $^{1,4,6}$.

Estos temas se debatían en el ambiente quirúrgico europeo, más precisamente el alemán, cuando el insigne cirujano chileno doctor Lucas Sierra Mendoza desarrollaba su especialización visitando los más afamados centros de cirugía en el Viejo Continente. De modo que, a su regreso, empapado de las nuevas técnicas efectuó tempranamente la primera colecistectomía en Chile cuando corría el año 1899.

\section{El doctor Lucas Sierra y su entorno quirúrgico}

La acertada decisión del decano José Joaquín Aguirre de enviar a Europa a formar especialistas chilenos, determinó que el doctor Manuel Barros Borgoño, a poco de su regreso en 1879 , tomara a su cargo una segunda Cátedra de Cirugía en el Hospital San Juan de Dios. Junto a Ventura Carvallo Elizalde, se iniciaría, entonces, una etapa de decisivo desarrollo de la cirugía chilena. Los principios de antisepsia comenzaron a penetrar en la mentalidad local y con ello la posibilidad de abordar quirúrgicamente las cavidades viscerales. Junto a Barros Borgoño creció Lucas Sierra, novel cirujano que también viajó a los principales centros europeos de la época y a su vuelta asumió como Jefe de Clínica9. Más adelante, fallecido su maestro, asumió la titularidad de la Cátedra de Clínica Quirúrgica. Previamente, cuando Carvallo se desplazó al naciente Hospital San Vicente de Paul, primer hospital clínico junto a la Facultad de Medicina de la Universidad de Chile, Sierra tomó la Cátedra en el Hospital San Borja ${ }^{10}$. De esta forma, el doctor Sierra operaba en la mañana en el Hospital Universitario de San Vicente y en la tarde en el San Borja. Más adelante, también tomó la Cátedra del Hospital del Salvador. Sierra fue un cirujano de grandes capacidades técnicas, organizativas y académicas, también humanas, lo que queda reflejado al señalar ${ }^{11}$ : "No interesan la técnica, ni la divagación clínica, ni el arte quirúrgico como cosas decisivas; lo fundamental será siempre la vida del enfermo" (Figura 2).

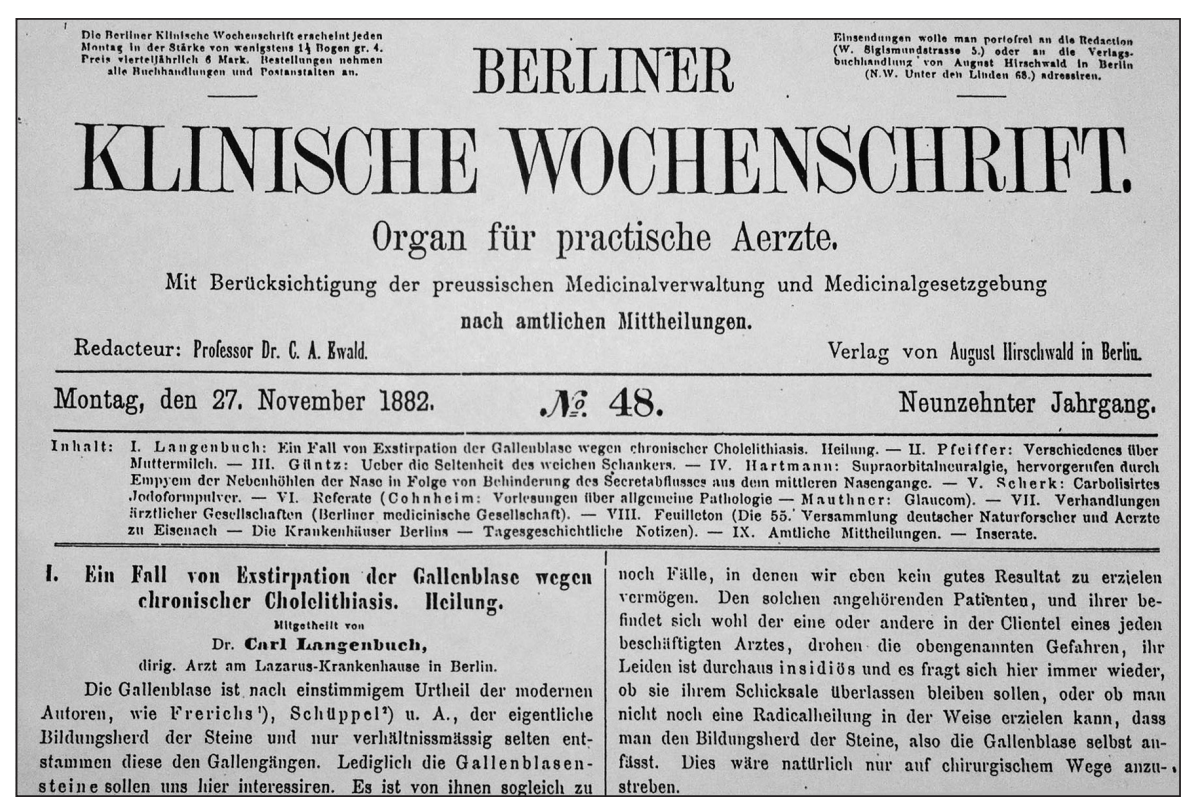

Figura 1. Fragmento del Berliner Klinische Wochenschrift, 1882, en donde Carl Langenbuch comunica su primera colecistectomía. 


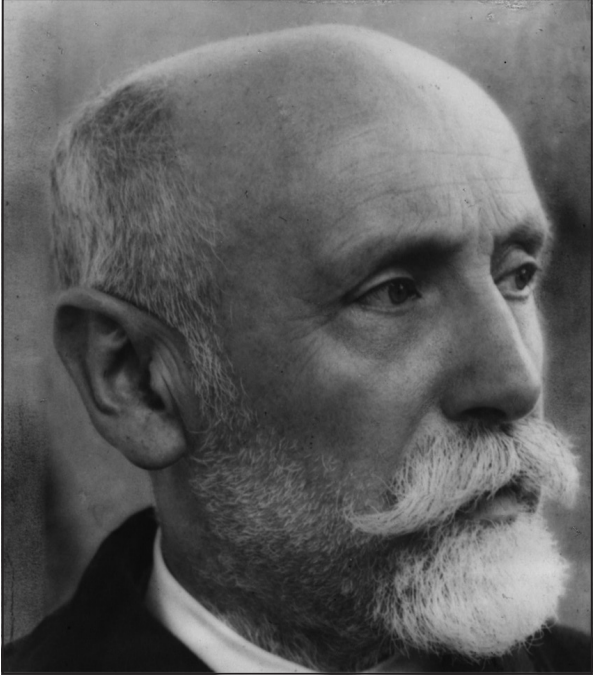

Figura 2. El Dr. Lucas Sierra Mendoza (1866-1937).

Lucas Sierra, como buen académico, fue un habitual colaborador de la Sociedad Médica de Santiago. La historia de esta Sociedad nos ha sido legada muy detalladamente por el doctor Camilo Larraín ${ }^{11}$ y a él recurriremos frecuentemente en este desarrollo. Fundada en 1869, esta Sociedad sesionaba semanalmente en su antiguo local en calle Huérfanos y durante décadas, cada viernes en la noche, concurrían entre 15 y 20 médicos y un grupo numeroso de estudiantes y se presentaban casos que cubrían todo el espectro de la medicina, aunque predominaban los temas quirúrgicos ${ }^{11}$. Incluso, muchas veces, se citaba a pacientes que podían ser examinados por los asistentes. Pues bien, en la sesión del 27 de octubre de 1899, el doctor Sierra, con la pieza quirúrgica en las manos, dio cuenta de la primera colecistectomía realizada en Chile, efectuada por él en el Hospital San Borja ${ }^{12}$. En palabras del doctor Sierra, se trataba de "una enferma de 45 años que sufría de cólicos hepáticos desde 13 años y que antes de ser operada tenía temperatura de hasta $39^{\circ}$ ". Agrega el acta de aquella sesión, que se prolongó hasta las 11PM, que el doctor Sierra expresara que la vesícula extirpada: "no estaba muy grande pero con sus paredes engrosadas y llenas de cálculos"12. Pero es más, la paciente también tenía cálculos en el colédoco, lo que lo motivó a explorarlo, extraer el cálculo que estaba alojado en su interior y a hacer la colédocorrafia correspondiente (en dos planos) y a dejar un drenaje. Esta exploración de colédoco en la primera colecistectomía determinó que presentase su caso quirúrgico en el siguiente Congreso de Cirugía en París ${ }^{10,11}$.

Algunos años más tarde, en un número de la
Revista Médica de Chile de 1916 y cuando ya tenía la experiencia de haber efectuado 200 colecistectomías, el doctor Sierra ${ }^{13}$, presentó un estudio comparativo sobre si en las enfermedades biliares, "calculosas o no", era preferible "la escisión de la Vesícula versus el desagüe". Eran los tiempos de los vapores del éter y la fenicación del ambiente quirúrgico, y en que los cirujanos ya se cubrían de un modo especial al momento de operar. Las palabras del historiador Larraín ${ }^{11}$, para describir una sala de cirugía general eran: "Alrededor de una vulgar mesa de madera que servía de mesa de operaciones se habían dispuesto una fuente pescadera en la que se hervían algunos instrumentos, un lavatorio, una tetera en la cual se traía el agua caliente, etc.; irrigadores de ácido fénico en profusión, unos cuantos paquetes de algodones impregnados con el mismo poderoso antiséptico completaban la dotación quirúrgica". Para la cirugía abdominal se disponía de otra sala "que fuera de estar a unos cuantos metros de altura i de permitir por esta circunstancia gozar del hermoso panorama que ofrece nuestra cordillera, no presentaba mayores garantías ni comodidades". Sólo a comienzos del 1900 se pudo contar con un autoclave, mesas quirúrgicas y "artefactos que hacían posible la esterilización del agua y de los demás utensilios indispensables en toda sala de operaciones".

En la interesante publicación de 1916, Sierra exponía con todo detalle su concepción etiopatogénica de las enfermedades biliares. Con mucha fuerza y convencimiento planteaba que en los problemas biliares calculosos, "su origen netamente infeccioso está hoy plenamente evidenciado" y que "los gérmenes infecciosos" llegan habitualmente por vía sanguínea. Añadía: "la defensa natural que presenta la vesícula puede hacer perfectamente que los microbios que han llegado hasta ella sucumban, la colesterina se deposita a su alrededor y les forma la tumba, o sea, el cálculo donde van a yacer sepultados" se hablaba, por tanto, de las "infecciones biliares calculosas" y así, cuando la "vesícula es infectada a dosis pequeñas (sub-infectada) va a dar síntomas prodromales o inaugurales". Estos síntomas los describe de la siguiente forma: Inmediatamente de ingerida cierta clase de alimentos, o dentro de los tres cuartos de hora que le siguen, (el paciente) experimenta una sensación de peso, de distensión o tirantez o repleción de la parte alta del abdomen, que le obliga a desabotonar las ropas, a doblar el cuerpo hacia adelante; si vomita cesa toda molestia", cuadro que no difiere de una vulgar “indigestión... sin pensar siquiera que están en presencia de una dispepsia biliar". Sigue describiendo el mismo Sierra: "Después de cada indigestión, nota el enfermo todo el costado derecho adolorido y tirante y en ocasiones, experimenta un dolor de 
cierta intensidad al querer respirar profundamente". Pero en otras ocasiones, si "aquellos microbios que van a ser distribuidos gradual y lentamente" o se encuentran "alteradas suficientemente las paredes de la vesícula por su acción continuada, o la llegada de una dosis maciza, estalla un ataque". Conforme continúe la infección, "se pasa al período de la obstrucción de la infección biliar calculosa" que no es "síntoma de una infección biliar calculosa sino una complicación; complicación tardía y grave, que los cirujanos queremos prevenir".

Pues bien, Sierra señalaba en la referida publicación $^{13}$ que para algunos, en la etapa inicial "la enfermedad pertenece al médico, otros que desde el principio al cirujano", pero asintiendo con Kocher "antes que a nadie, al enfermo". Agrega Sierra: "Jamás debemos olvidar que curar el síntoma o el ataque no es de ninguna manera curar la enfermedad" y por lo tanto, "el tratamiento médico es ilusorio, paliativo; no sana al enfermo". En su experiencia, tras una colecistectomía "los enfermos han estado en condiciones de volver a sus casas a los 8 días". Por tanto, "está autorizado el cirujano para garantizar a estos enfermos que la operación puede curarlos de una manera más completa, segura y rápida que ningún otro medio, evitando o previniendo los ulteriores peligros que les amenazan".

También Sierra ${ }^{13}$, afirmaba lo dicho por $\mathrm{WJ}$ Mayo, en el sentido que "todos los cánceres de la vesícula se observan en antiguos colelitiásicos".

Por su participación en los principales hospitales del país, el doctor Sierra desarrolló una notable experiencia, que no sólo se centró en la cirugía abdominal, donde abordó temas biliares, apendiculares y ulcerosos, sino que también efectuó cirugía ginecológica y del cuello. En palabras de Larraín ${ }^{11}$ : "Sin duda el Profesor Sierra fue la figura dominante en la medicina chilena de la primera mitad del siglo XX y posiblemente de toda la centuria”. Presidió la Sociedad Médica de Santiago entre 1916 y 1918 y fue electo en 1922 como el primer Presidente de la Sociedad de Cirugía de Chile, precursora de la actual Sociedad de Cirujanos de Chile ${ }^{14}$. Formó generaciones de cirujanos para el naciente siglo XX. (Figura 3) Sus dotes personales también han sido noblemente reconocidos ${ }^{15}$.

\section{Desarrollo en los siglos posteriores}

Prácticamente sin mayores modificaciones, y en palabras de Sierra ${ }^{13}$, la ectomía vesicular efectuada por "Langenbuch en 1882, la que yo vi ejecutar a Mikulicz en 1895 y que en 1899 me cupo el honor por primera vez en Chile" siguió efectuándose sin mayores modificaciones, por décadas. Hacia 1920 ya se había establecido definitivamente, a nivel mundial, que la colecistectomía era el tratamiento del paciente con colelitiasis. El acto operatorio se perfeccionó con nuevas técnicas anestésicas y antisépticas, nuevos materiales de sutura y antibióticos, haciendo de la colecistectomía una cirugía segura, de baja morbilidad y escasa mortalidad.

La gran modificación de abordaje correspondió

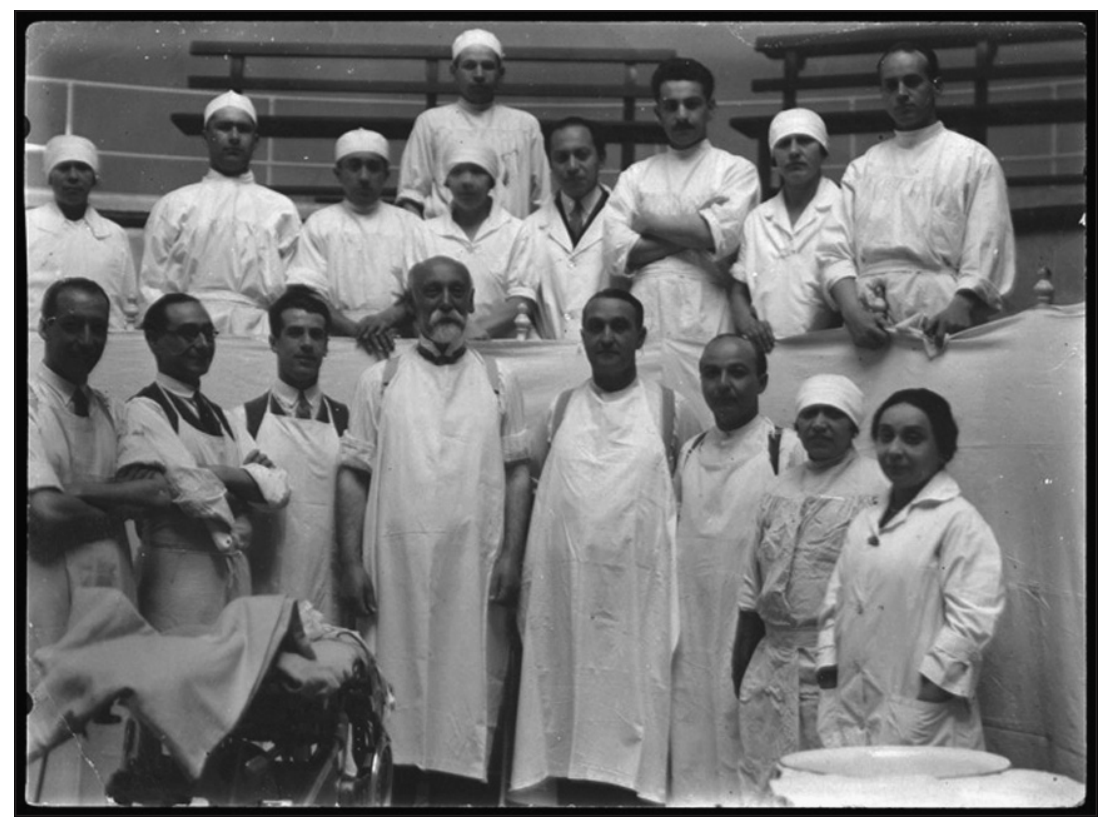

Figura 3. El doctor Lucas Sierra rodeado de ayudantes y discípulos en un anfiteatro quirúrgico. 
a la implementación de la laparoscopia moderna, a partir de los aportes de Muhe, Mouret y DuBois en Europa y Reddick en los Estados Unidos de Norteamérica ${ }^{7}$. Nuevos avances técnicos han permitido efectuar la colecistectomía vía laparoscópica con la introducción de sólo un trócar y más recientemente, por medio de la técnica NOTES (Natural Orifice Transluminal Endoscopic Surgery).

En Chile, en la serie de Hepp ${ }^{16}$, sobre 35.014 colecistectomías, la mortalidad fue de sólo un 0,04\% y las complicaciones de un 3,8\%. La hospitalización por una colecistectomía se ha reducido a un día o menos y la capacidad de retomar completamente las actividades a una semana. Se estima que en Chile (MINSAL, 2009) se efectúan unas 60.000 colecistectomías cada año, el $80 \%$ vía laparoscópica.

Queda pendiente desentrañar la patogenia de la enfermedad biliar litiásica, una deuda científica que mientras no esté saldada, y con ello la prevención, los cirujanos continuaremos repitiendo lo que nos señalara el doctor Lucas Sierra ${ }^{13}$ : "Las enfermedades (litiásicas) biliares son netamente quirúrgicas, no médicas".

\section{Referencias}

1. De U. Evolution of cholecystectomy: A tribute to Carl August Langenbuch. Indian J Surg 2004;66:97-100.

2. Hardy KJ. Carl Langenbuch and the Lazarus Hospital: events and circumstances surrounding the first cholecystectomy. Aust N Z J Surg. 1993;63:56-64.

3. Morgenstern L. Carl Langenbuch an the first cholecystectomy. Surg Endosc. 1992;6:113-4.
4. Traverso L W. Carl Langenbuch and the First Cholecystectomy. Am J Surg. 1978;132:81-2.

5. Langenbuch C. Ein fall von Exstirpation der Gallenblase wegen chronischer Cholelithiasis. Berlin Klin Wochenschr 1882;48:725-7.

6. Halpert B. Carl Langenbuch "Master Surgeon of the Biliary System" 1846-1901. Arch Surg. 1932;25:17882.

7. Soper NJ. Cholecystectomy: From Langenbuch to Natural Orifice Transluminal Endoscopic Surgery. World J Surg. 2011;35:1422-7.

8. De la Garza-Villaseñor L. Aspectos históricos de la anatomía quirúrgica de las vías biliares y la colecistectomía. Rev Gastroenterol Mex. 2001;66:210-4.

9. González I. Bosquejo histórico de la Enseñanza de la Cirugía en Chile. Arch Soc Cir Chile 1973;XXV:14563.

10. Puente S. Historia de la cirugía en el Hospital San Francisco de Borja. Rev Chil Cir. 2006;6:473-8.

11. Larraín Aguirre C. La Sociedad Médica de Santiago y el Desarrollo Histórico de la Medicina en Chile. Imprenta Salesianos. Chile, 2002.

12. Sierra L. Cálculos Biliares, Extirpación de la Vesícula. Rev Med Chile 1900;28:314.

13. Sierra L. Infecciones biliares. Calculosas o no. Escisión de la Vesícula versus desagüe. Rev Med Chile 1916;44:534:50.

14. Cubillos L. Historia de la Sociedad de Cirujanos de Chile. Primera parte. Rev Chil Cir. 2012; 64: 312-8

15. Neghme A. En el centenario del nacimiento del Profesor Dr. Lucas Sierra. Rev Med Chile 1967;95:278-80.

16. Hepp J, Ríos H. Colecistectomía laparoscópica: Evaluación de 35.014 operaciones realizadas en Chile. Rev Chil Cir. 1996;48:461-6. 